\title{
MUTUAL ABSOLUTE CONTINUITY OF SETS OF MEASURES
}

\begin{abstract}
BERTRAM WALSH ${ }^{1}$
Abstract. A theorem slightly stronger than the following is proved: If $K$ is a convex set of (signed) measures that are absolutely continuous with respect to some fixed positive sigma-finite measure, then the subset consisting of those measures in $K$ with respect to which all measures in $K$ are absolutely continuous is the complement of a set of first category in any topology finer than the norm topology of measures. This implies, e.g., that any Banachspace-valued measure $\mu$ is absolutely continuous with respect to $\left|\left\langle\mu(\cdot), x^{\prime}\right\rangle\right|$ for a norm-dense $G_{\delta}$ of elements $x^{\prime}$ of the dual of the Banach space.
\end{abstract}

Recently Rybakov [4] proved, by a direct but rather involved construction, that if $(S, \Sigma)$ is a measurable space and $\mu: \Sigma \rightarrow E$ a countably additive measure with values in a Banach space $E$, then there exists a linear functional $x_{0}^{\prime} \in E^{\prime}$ such that $\mu$ is absolutely continuous with respect to the (variation of the) scalar-valued measure $\left\langle\mu(\cdot), x_{0}^{\prime}\right\rangle$. This note gives a rather less involved proof of a stronger result, as indicated in the abstract above.

To fix our ideas and notation, let $(S, \Sigma, \lambda)$ be a $\sigma$-finite measure space; since there is a strictly positive function in $\mathscr{L}^{1}(\lambda)$, there is no loss of generality in taking $\lambda$ finite, which we shall do. We shall denote by $P_{n}(n=1,2, \cdots, \infty)$ the set of all $n$-tuples $\alpha=\left(\alpha_{1}, \alpha_{2}, \cdots\right)$ in $R^{n}$ (or $l_{R}^{1}$ if $\left.n=\infty\right)$ for which all $\alpha_{i}>0$, and by $Q_{n}(n=1,2, \cdots, \infty)$ the subset of $P_{n}$ for which $\sum_{i} \alpha_{i}=1$. It is obvious that the $P_{n}$ 's and $Q_{n}$ 's are $G_{\delta}^{\prime}$ 's in their ambient spaces $R^{n}$ and $l_{R}^{1}$, and thus their topologies can be generated by complete metrics; in particular, they are Baire spaces (spaces for which the Baire category theorem holds).

Any measure-theoretic terms or notation not otherwise explained agree with the usage of [1]-except that $|\mu|$ is the variation of $\mu$.

Lemma 1. For any set $C \subseteq c a(S, \Sigma)$ of measures absolutely continuous with respect to $\lambda$, there exists a countable set $\left\{\mu_{i}\right\}_{i \in I} \subseteq C$ such that if $\left|\mu_{i}\right|(A)=0$ for all $i$, then $|\mu|(A)=0$ for all $\mu \in C$.

Received by the editors September 24, 1970.

AMS 1970 subject classifications. Primary 28A10, 28A45, 46G10; Secondary 47B99.

Key words and phrases. Measures, absolute continuity, vector-valued measures. Choquet simplex, maximal measures.

${ }^{1}$ Partially supported by NSF-GP-21182.

Copyright (c) 1971, American Mathematical Society 
Proof. Assuming $C \neq \varnothing \varnothing$, one can find sets $A \in \Sigma$ for which there exist elements $\mu \in C$ such that $|\mu|(A)>0$ and every $|\mu|$-null subset of $A$ is $\lambda$-null; e.g., if $\mu \neq 0$ and $f$ is its Radon-Nikodým derivative with respect to $\lambda$, then $A=\{s \in S:|f(s)| \neq 0\}$ has that property. Zorn's lemma gives a maximal disjoint family $\left\{A_{i}\right\}_{i \in I}$ of such sets, with corresponding measures $\left\{\mu_{i}\right\}_{i \in I}$. Since all but countably many $A_{i}$ 's must be $\lambda$-null, $I$ is countable; the existence of an $A \in \Sigma$ with $\left|\mu_{i}\right|(A)=0$ for all $i \in I$ but $|\mu|(A)>0$ for some $\mu \in C$ would clearly contradict maximality. Q.E.D.

Let $\mathfrak{T}$ denote the topological vector space of all $\Sigma$-measurable scalar-valued functions on $S$ under the topology of convergence in $\lambda$ measure. It is easy to verify that for any $A \in \Sigma, \delta>0$ and open set $U$ of scalars, the set $\{f \in \mathscr{M}: \lambda(\{s \in A: f(s) \notin U\})<\delta\}$ is an open set in $\mathfrak{T}$, and (consequently) the set $\{f \in \mathfrak{M}: \lambda(\{s \in A: f(s) \notin U\})=0\}$ is a $G_{\delta}$ in $\mathfrak{T}$.

Lемма 2. Let $\left\{f_{i}\right\}_{i=1}^{\infty}$ be a sequence in $\mathfrak{T}$, and set $B_{i}=$ $\left\{s \in S: f_{i}(s) \neq 0\right\}$. Then

(1) for any finite $n$, the set of $\alpha \in P_{n}$ or $Q_{n}$ for which

$$
\lambda\left(\left\{s \in \bigcup_{i=1}^{n} B_{i}:\left|\sum_{i=1}^{n} \alpha_{i} f_{i}(s)\right|=0\right\}\right)=0
$$

is a dense $G_{\delta}$ in $P_{n}$ or $Q_{n}$ respectively; moreover,

(2) the set of $\alpha \in Q_{\infty}$ for which

$$
\lambda\left(\left\{s \in \bigcup_{i=1}^{n} B_{i}:\left|\sum_{i=1}^{n} \alpha_{i} f_{i}(s)\right|=0\right\}\right)=0
$$

for all finite $n$ is a dense $G_{\delta}$ in $Q_{\infty}$.

Proof. For any finite $n$, the map $\left(\alpha_{1}, \alpha_{2}, \cdots\right) \rightarrow \sum_{i=1}^{n} \alpha_{i} f_{i}$ is continuous from $R^{n}$ or $l_{R}^{1}$ to $\mathfrak{T}$, and thus all the sets in question are $G_{\delta}$ 's. To prove the "density" part of assertion (1) for $Q_{n}$ it clearly suffices to prove the part concerning $P_{n}$, and because $P_{n}=P_{n-1} \times P_{1}$ a proof by induction on $n$ follows immediately from verification of the case $n=2$. To check that case we need only observe that the sets $S_{\beta}=\left\{s \in B_{1} \cup B_{2}: f_{1}(s)+\beta f_{2}(s)=0\right\}$ are disjoint for distinct $\beta \in P_{1}$, and must therefore all be $\lambda$-null except for countably many choices of $\beta$. It follows that if $\left(\alpha_{1}, \alpha_{2}\right)$ does not belong to the nowhere-dense set of solutions of $\alpha_{2}=\beta \alpha_{1}$ for one of those countably many $\beta$ 's, then

$$
\lambda\left(\left\{s \in B_{1} \cup B_{2}: \alpha_{1} f_{1}(s)+\alpha_{2} f_{2}(s)=0\right\}\right)=0 ;
$$


so the set of $\left(\alpha_{1}, \alpha_{2}\right)$ 's for which that relation holds contains the complement of a set of first category and is thus dense.

From assertion (1) it follows immediately that for any fixed finite $n$ the set

$$
\left\{\alpha \in Q_{\infty}: \lambda\left(\left\{s \in \bigcup_{i=1}^{n} B_{i}: \sum_{i=1}^{n} \alpha_{i} f_{i}(s)=0\right\}\right)=0\right\}
$$

is a dense $G_{\delta}$ in $Q_{\infty}$. The set of $\alpha \in Q_{\infty}$ for which that relation holds for all finite $n$ is thus a countable intersection of dense $G_{\delta}$ 's in $Q_{\infty}$ and thus again dense by the Baire category theorem, a fact which establishes (2). Q.E.D.

We can now prove the principal theorem, stated in somewhat more general form than given in the abstract above.

THEOREM. Let $K$ be a bounded ${ }^{2}$ convex set in a locally convex space $F$, and suppose $K$ is a Baire space. Let $T: K \rightarrow c a(S, \Sigma)$ be an affine map continuous in the norm topology of ca $(S, \Sigma)$, and suppose that the measures in $T[K]$ are all absolutely continuous with respect to $\lambda$. Then the set of points $x \in K$ for which all measures in $T[K]$ are absolutely continuous with respect to $|T x|$ is a dense $G_{\delta}$ in $K$.

Proof. Setting $C=T[K]$ in Lemma 1 , we can select a sequence $\left\{x_{i}\right\}_{i=2}^{\infty}$ in $K$ which has the property that if $\mu_{i}=T x_{i}$, then $\left|\mu_{i}\right|(A)=0$ for all $i$ implies $|T x|(A)=0$ for all $x \in K$. Let $f_{i}$ be a Radon-Nikodým derivative of $\mu_{i}$ with respect to $\lambda, i=2,3, \cdots$, let $B_{i}$ $=\left\{s \in S: f_{i}(s) \neq 0\right\}$, and let $B=\bigcup_{i=2}^{\infty} B_{i}$. For each $\epsilon>0$, the set

$W_{\epsilon}=\{\nu \in c a(S, \Sigma):$ there exists $\delta>0$ such that

$$
|\nu|(A)<\delta \text { implies } \lambda(A \cap B)<\epsilon\}
$$

is open in the norm topology of $c a(S, \Sigma)$; indeed, if $\delta$ has that property for $\nu$ and $\|\nu-\pi\|<\delta / 2$, then $\delta / 2$ has that property for $\pi$. Thus $T^{-1}\left[W_{\epsilon}\right]=\{x \in K$ :there exists $\delta>0$ such that $|T x|(A)<\delta$ implies $\lambda(A \cap B)<\epsilon\}$ is open in $K$, and we claim it is dense. To see this, let a point $x_{1} \in K$ and a convex zero-neighborhood $N$ in $F$ be given, and let $f_{1}$ be a Radon-Nikodým derivative of $\mu_{1}=T x_{1}$; set $B_{1}=\left\{s \in S: f_{1}(s) \neq 0\right\}$. Let $\eta>0$ be so small that $0<t<\eta \Rightarrow(1-t) x_{1}$ $+t K \subseteq x_{1}+N$; this is possible since $K$ is bounded. Applying Lemma 2 to the sequence $\left\{f_{i}\right\}_{i=1}^{\infty}$, we can find an $\alpha \in Q_{\infty}$ satisfying condition (2) of that lemma and such that $\sum_{i=2}^{\infty} \alpha_{i}<\eta$. If $n$ is so large that $\left(\sum_{i=1}^{n} \alpha_{i}\right)^{-1}\left(\sum_{i=2}^{n} \alpha_{i}\right)<\eta$ and $\lambda\left(B \backslash \bigcup_{i=2}^{n} B_{i}\right)<\epsilon / 2$, then setting $\gamma_{i}$

${ }^{2}$ Alternatives to this hypothesis are possible. E.g., the assertion of the theorem holds if $K$ is metrizable and $0 \in K$, even if $K$ is unbounded. 
$=\left(\sum_{i=1}^{n} \alpha_{i}\right)^{-1} \alpha_{i}$ we have $\sum_{i=1}^{n} \gamma_{i} x_{i} \in K \cap\left(x_{1}+N\right)$, and $T\left(\sum_{i=1}^{n} \gamma_{i} x_{i}\right)$ $=\sum_{i=1}^{n} \gamma_{i} \mu_{i}$ which has Radon-Nikodým derivative $\sum_{i=1}^{n} \gamma_{i} f_{i}$ with respect to $\lambda$. Since

$$
\lambda\left(\left\{s \in \bigcup_{i=1}^{n} B_{i}: \sum_{i=1}^{n} \gamma_{i} f_{i}(s)=0\right\}\right)=0
$$

(for the $\gamma_{i}^{\prime}$ 's are proportional to the $\alpha_{i}^{\prime}$ 's), one sees that $\lambda$ is absolutely continuous with respect to $\left|\sum_{i=1}^{n} \gamma_{i} \mu_{i}\right|$ on $\bigcup_{i=2}^{n} B_{i}$, i.e., if $H \subseteq \bigcup_{i=2}^{n} B_{i}$ then $\lambda(H)$ can be made arbitrarily small by taking $\left|\sum_{i=1}^{n} \gamma_{i} \mu_{i}\right|(H)$ sufficiently small. In particular, there exists $\delta>0$ with the property that for such an $H,\left|\sum_{i=1}^{n} \gamma_{i} \mu_{i}\right|(H)<\delta \Rightarrow \lambda(H)<\epsilon / 2$; so if $A \in \Sigma$ is such that $\left|\sum_{i=1}^{n} \gamma_{i} \mu_{i}\right|(A)<\delta$, then

$$
\lambda\left(A \cap \bigcup_{i=2}^{n} B_{i}\right)<\epsilon / 2 \text { and } \lambda\left(A \cap\left(B \backslash \bigcup_{i=2}^{n} B_{i}\right)\right)<\epsilon / 2
$$

and thus $\lambda(A \cap B)<\epsilon$.

We now need only use the fact that $K$ is a Baire space to conclude that $K_{0}=T^{-1}\left[\cap_{k=1}^{\infty} W_{1 / k}\right]$ is dense in $K$. It is immediate that $K_{0}$ is the set of all $x \in K$ such that $|T x|(A)=0$ implies $\lambda(A \cap B)=0$; but since $S \backslash B$ is null with respect to all $\left\{\mu_{i}\right\}_{i=2}^{\infty}$ and thus with respect to $|T y|$ for all $y \in K, K_{0}$ is the set of all $x \in K$ such that $|T x|(A)$ $=0 \Rightarrow|T y|(A)=0$ for all $y \in K$. Q.E.D.

A strengthened form of Rybakov's theorem follows at once. Indeed, if $\mu: \Sigma \rightarrow E$ is a Banach-space valued vector measure, there is an adjoint linear mapping $T: E^{\prime} \rightarrow c a(S, \Sigma)$ given by $\left(T x^{\prime}\right)(A)=$ $\left\langle\mu(A), x^{\prime}\right\rangle$ and $T$ is continuous in the norm topologies. It is known that there exists a finite positive measure $\lambda \in c a(S, \Sigma)$ with respect to which all the measures $T x^{\prime}$ are absolutely continuous (see [1, IV.10.5, p. 321] or, for a direct, elegant proof see [2, Theorem 3.10, p. $199 \mathrm{ff}]$.$) . Taking the set K$ of our theorem to be the closed unit ball in $E^{\prime}$ (with the norm topology) and $T$ to be the mapping just described, we see that the set $K_{0}$ of $x_{0}^{\prime}$ in that unit ball having the property that $\mu$ is absolutely continuous with respect to $\left\langle\mu(\cdot), x_{0}^{\prime}\right\rangle$ is a norm-dense $G_{\delta}$. This has the amusing consequence that the norm of elements $x \in E$ can be computed by taking the supremum of $\left|\left\langle x, x_{0}^{\prime}\right\rangle\right|$ over $x_{0}^{\prime} \in K_{0}$. For another application of the theorem, suppose $X$ is a simplex in the sense of Choquet [3], and let $P$ be the unique linear extension of the mapping which assigns to each positive measure $\mu$ on $X$ the maximal measure which represents it. $P$ is norm-decreasing and therefore norm-continuous. Thus if $K$ is a norm-closed convex set of measures on $X$ such that $P[K]$ is ab- 
solutely continuous with respect to some fixed positive measure $\lambda$, then there is a norm-dense $G_{\delta}$ set $K_{0} \subseteq K$ such that every measure in $P[K]$ is absolutely continuous with respect to $|P \mu|$ for each $\mu \in K_{0}$.

\section{REFERENCES}

1. N. Dunford and J. T. Schwartz, Linear operators. I: General theory, Pure and Appl. Math., vol. 7, Interscience, New York, 1958. MR 22 \#8302.

2. G. G. Gould, Integration over vector-valued measures, Proc. London Math. Soc. (3) 15 (1965), 193-225. MR 30 \#484.

3. R. R. Phelps, Lectures on Choquet's theorem, Van Nostrand, Princeton, N. J., 1966. MR $33 \# 1690$.

4. V. I. Rybakov, $K$ teoreme Bartla-Danforda-Šarca o vektornyh merah, Mat. Zametki 7 (1970), 247-254.

Rutgers University, New Brunswick, New Jersey 08903 